\title{
Analytical Examination of Oxidized Free and Esterified 7-Ketocholesterol and Related Oxysterols in Human Plasma Incubated with Copper
}

\author{
Naoki Tamasawa and Kazuo Takebe \\ The Third Department of Internal Medicine, Hirosaki \\ University School of Medicine, Hirosaki 036
}

\begin{abstract}
Tamasawa, N. and Takebe, K. Analytical Examination of Oxidized Free and Esterified 7-Ketocholesterol and Related Oxysterols in Human Plasma Incubated with Copper. Tohoku J. Exp. Med., 1992, 168 (1), 37-45 - Oxygenated derivatives of cholesterol (oxysterols) have been demonstrated to possess a wide variety of biological properties and evaluated for their abilities to inhibit cholesterol biosynthesis. We investigated a method to analyze copper-catalyzed oxidation products of human plasma cholesterol. Free and esterified oxysterols produced were mainly 7-ketocholesterol, and small amounts of $7 \beta$-hydroxycholesterol and 5, $6 \alpha$-epoxy-cholesterol were also identified. Quantitatively, the sterol nucleus of ester was less susceptible to oxidation than that of the free form. This finding suggested that the cholesterol nucleus of ester form was more resistant against oxidative stress than free form. Additionally we demonstrated that the addition of probucol, a powerful antioxidant used clinically to lower blood cholesterol, inhibited this copper-catalyzed oxdiation of cholesterol. - 7ketocholesterol ; free oxysterol; esterified oxysterol ; cholesterol oxdiation; probucol
\end{abstract}

Oxidatively modified low density lipoprotein (LDL), which is the major carrier of plasma cholesterol may be entrapped by macrophage through a scavenger pathway and contribute to foam cell formation during atherosclerosis (Brown and Goldstein 1983; Steinbrecher et al. 1984 ; Lenz et al. 1990). Nevertheless, oxidative modification of the lipid component of lipoprotein especially cholesterol has not yet been investigated sufficiently. Oxygenated derivatives of cholesterol, oxysterols, have been demonstrated to possess a variety of biological properties, including cytotoxicity, atherogenicity, mutagenicity and cartinogenicity (Smith 1981). Several oxysterols are powerful inhibitors of cholesterol biosynthesis (Kandutsch et al. 1978). This inhibitory effect appears to be due to a suppression of the activity of 3-hydroxy-3-methylglutaryl (HMG)-CoA reductase (Brown and Goldstein 1974). We submitted human plasma to copper-catalyzed oxidation then separated free and esterified (fatty-acylated) oxysterols separately and measured them quantitatively by gasliquid chromatography (GLC). We also

Received July 20, 1992 ; revision accepted for publication September 4, 1992. 
investigated the inhibitory effect of probucol on that oxidation.

\section{Materials and Methods}

\section{Chemicals}

All chemicals used were of analytical grade. $5 \beta$-cholestane, $3 \beta$-hydroxycholest-5-ene-7one (7-ketocholesterol), cholest-5ene- $3 \beta, 7 \beta$-diol $(7 \beta$-OH-chol) and $5,6 \alpha$-epoxy-5 $\alpha$ cholestan-3 $\beta$-ol (5, $6 \alpha$-epoxy-chol) were purchased from Sigma Chemical Co. (St. Louis, MO, USA).

Palmityl ester of 7-ketocholesterol was synthesized using cholesterol esterase (sterolester acylhydrolase [EC 3. 11. 13], Sigma) (Billheimer et al. 1983). 7-Ketocholesterol (25 $\mu \mathrm{mol})$ and palmitate $(50 \mu \mathrm{mol})$ were dried on the bottom of a conical tube. Petroleum ether $(100 \mu \mathrm{l})$ and a solution $(5.6 \mathrm{ml})$ containing sodium taurocholate $(8.9 \mathrm{mM})$ in $0.05 \mathrm{M}$ phosphate buffer, $\mathrm{pH}$ 6.0, were added. After sonication, 6.25 units of cholesterol esterase was added and the mixture was incubated for $48 \mathrm{hr}$ at $37^{\circ} \mathrm{C}$. Lipids were extracted with $20 \mathrm{ml}$ of chloroform/methanol $(2: 1, \mathrm{v} / \mathrm{v})$. Thin-layer chromatography (TLC) was performed using a silica 60 pre-coated TLC plate (Merk, Darmstadt, Germany) with the solvent system of hexane/diethyl ether/acetic acid $(20: 10: 1, \mathrm{v} / \mathrm{v} / \mathrm{v})$. A single spot of the synthesized oxysteryl ester on the TLC plate (Rf value 0.73 ) was observed between cholesteryl palmitate (1.00) and free cholesterol (0.23).

Probucol was kindly supplied by Otsuka Pharmaceuticals Ltd. (Tokyo).

\section{Extraction and separation of oxysterol}

Heparinized blood specimens were obtained from healthy human subjects just before the experiment. Plasma lipids were extracted by Folch method, and the separation of oxysterols was carried out using a silica column (Fig. 1). Four volume of chloroform/

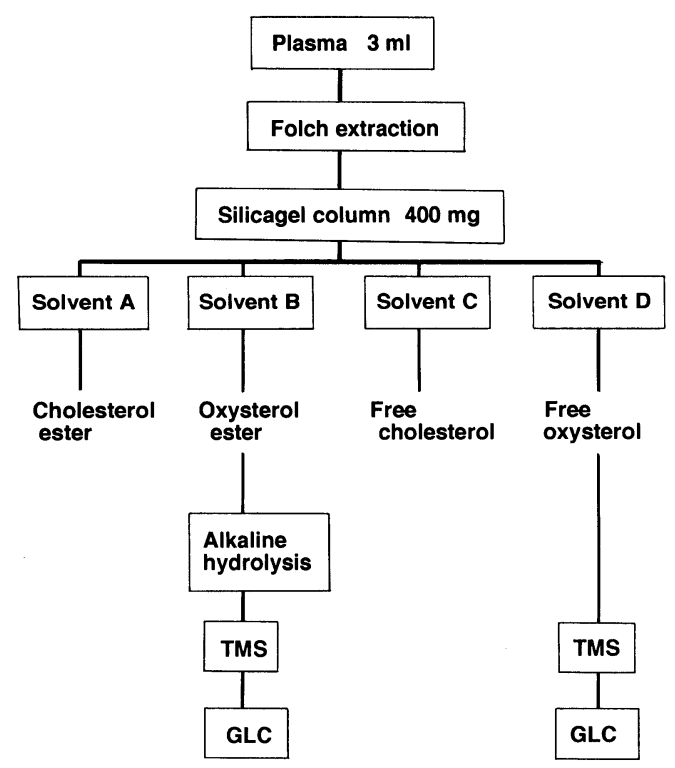

Fig. 1. Outline of the procedure for the analysis of plasma free and esterified oxysterol. Solvents A, B, C, and D were as depicted in Table 1.

TMS, trimethylsilyl derivatization; GLC, gas-liquid chromatography. 
TABLE 1. Solvent systems for the separation of cholesterol and oxysterol on a silica column ${ }^{\mathrm{a}}$

\begin{tabular}{lll}
\hline & \multicolumn{1}{c}{ Composition } & Compound eluted \\
\hline Solvent A & Hexane/Dichloromethane $(4: 1)$ & Cholesterol ester \\
Solvent B & Hexane/Dichloromethane $(1: 4)$ & Oxysterol ester \\
Solvent C & Dichloromethane & Free cholesterol \\
Solvent D & Ethylacetate & Free oxysterol \\
\hline
\end{tabular}

aFor the quantitative analysis of low levels of oxysterols, it is necessary to separated them from free and esterified cholosterol. Therefore, after the sample was applied to a silica column ( $400 \mathrm{mg}$ ) with $4 \mathrm{ml}$ of solvent A, the column was eluted successively with solvent $\mathrm{A}(8 \mathrm{ml})$, solvent $\mathrm{B}(8 \mathrm{ml})$, dichloromethane $(8 \mathrm{ml})$, and ethylacetate $(5 \mathrm{ml})$.

methanol $(2: 1, \mathrm{v} / \mathrm{v})$ solution was added to $3 \mathrm{ml}$ of plasma. After washing with 0.2 volume of water, organic phase was dried under a stream of nitrogen. The dried material was dissolved in $4 \mathrm{ml}$ of hexan/dichloromethane $(4: 1, \mathrm{v} / \mathrm{v})$ solution and applied onto a packed silica column (400 mg; Silicagel, Gasukurokogyo, Tokyo). For the quantitative analysis of oxysterols by GLC, it was necessary to clearly separate them from free and esterified cholesterol. This was achieved by eluting cholesteryl ester, oxysteryl ester, free cholesterol and free oxysterol in order from the silica column using solvents A, B, C, and D, respectively (Table 1). Oxysteryl ester was dried under nitrogen and subjected to mild alkaline hydrolysis in a methanol solution containing $4 \% \mathrm{KOH}$ at $50^{\circ} \mathrm{C}$ for $60 \mathrm{~min}$. Hydrolysis was confirmed by a TLC with hexan/diethyl ether/acetic acid $(20: 10: 1, \mathrm{v} / \mathrm{v} / \mathrm{v})$. Oxysterol was deri- vatized to trimethylsilyl (TMS) ether with TMS reagent (SIGMA-SIL-A, Sigma), and analyzed by using a Shimadzu gas chromatograph GC-8A equipped with a Shimadzu capillary column Hi-Cap coated with a cross-linked methyl-silicon $(25 \mathrm{~m} \times 0.32 \mathrm{~mm}$ i.d.) at an oven temperature of $260^{\circ} \mathrm{C}$. Peaks were identified using authentic TMS derivatized compounds on the basis of retention time and measured with an integrator-calculator (Shimadzu Chromatopac C-R3A, Shimadzu, Kyoto). Retention time of each compound relative to $5 \beta$-cholestane $(1.00)$ was as follows : cholesterol, $2.01 ; 7 \beta$-hydroxy-cholesterol, $2.70 ; 5,6 \alpha$-epoxy-cholesterol, 2.78 ; and 7-ketocholesterol, 3.78.

\section{Copper-catalyzed oxidation of plasma cholesterol}

Cupric sulfate in $0.9 \% \mathrm{NaCl}$ was added to plasma $(3 \mathrm{ml})$ and the final concentration was adjusted to $0,0.5,1.0$ or $2.0 \mathrm{mM}$. Then incubation was carried out on a shaking bath for $24 \mathrm{hr}$ at $37^{\circ} \mathrm{C}$. After the incubation, oxysterols were extracted and separated as described above.

\section{Antioxidant effect of probucol}

Probucol dissolved in ethanol was added to $3 \mathrm{ml}$ of plasma to make a final concentration of $227 \mu \mathrm{M}$. Then copper ion was added to a final concentration of $2 \mathrm{mM}$. After the incubation under the same condition, oxysterols were analyzed and compared with plasma incubated with copper $(2.0 \mathrm{mM})$ in the absence of probucol.

\section{Plasma levels of cholesterol}

Plasma total and free cholesterol levels were measured by an enzymatic method using a commercially available reagent kit (C-test, Wako, Osaka). Cholesteryl ester level was calculated by subtracting the free level from the total. 


\section{Statistics}

The data was expressed as the mean \pm s.D. Statistical significance was determined by Wilcoxon rank-sum test.

\section{RESULTS}

Separation and recovery of free and esterified oxysterol

The separation on a silica column of free and esterified oxysterols from cholesterols is shown in Fig. 2. 7-Keto-cholesteryl palmitate was clearly separated from cholesteryl oleate, a common plasma cholesteryl ester. As shown in Fig. 2 , the effluent eluted with the solvent B contained a small amount of free cholesterol. This fact may warrant the elution of other oxysterol esters such as $7 \alpha$-hydroxy-, $7 \beta$-hydroxy-and 5, $6 \alpha$-epoxy-cholesterol in this fraction (Assmann et al. 1975). The effluent eluted with the solvent $\mathrm{C}$ washed out free cholesterol from the column almost completely. Then, in the effluent eluted with the solvent $\mathrm{D}$, almost all of 7-ketocholesterol was eluted, This fraction may also contain other oxysterols, if any, because very polar taurocholate (taurine conjugated $3 \alpha$, $7 \alpha 12 \alpha$-trihydroxy cholanoic acid) was eluted in this fraction (data was not shown).

The recoveries of free 7-ketocholesterol and its palmityl ester added to plasma $(n=3)$ were $71.1 \% \pm 3.9 \%$ and $53.8 \% \pm 5.2 \%$, respectively.

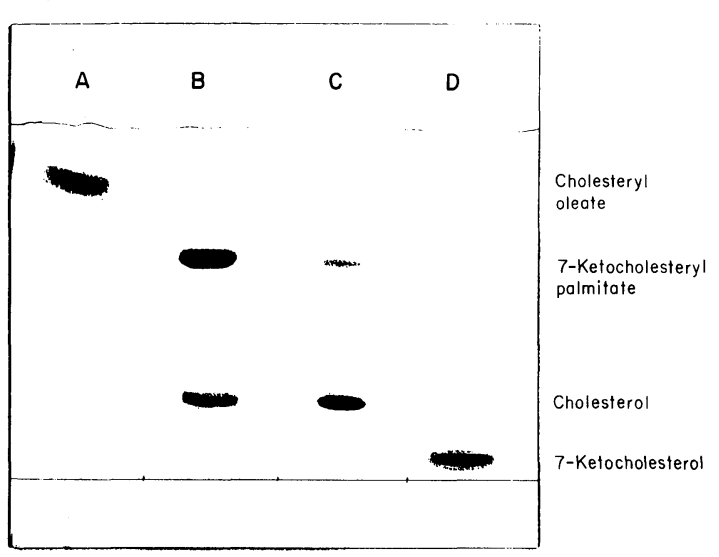

Fig. 2. Thin-layer chromatography to confirm the separation of cholesterol and its derivatives on a silica column. A mixture solution containing free cholesterol, 7-ketocholesterol, cholesteryl oleate, and 7-ketocholesteryl palmitate was applied on a silica column, and then each effluent with solvents A, B, C, and D (Table 1) was developed on a TLC plate (pre-coated TLC plate silica 60, Merk) using hexan/diethyl ether/acetic acid (20:10:1, v/v/v). Spots were detected with $\mathrm{I}_{2}$. 
Measurement of oxysterol levels in oxidized human plasma

Gas chromatograms derived from free oxysterol fraction are depicted in Fig. 3. Lower chromatogram (Fig. 3-B), which was obtained with plasma sample incubated in the presence of $2 \mathrm{mM} \mathrm{CuSO}_{4}$, demonstrated 7-ketocholesterol, as the major oxysterol generated (Jialal et al. 1991). A small amount of $7 \beta$-hydroxyand 5, $6 \alpha$-epoxy-cholesterol were also identified. In addition some other unidentified peaks were demonstrated; however, the sensitivity of the method did not allow quantitative determination of oxysterol other than 7-ketocholesterol. On the other hand, no significant peak excepting trivial 7-ketocholesterol was identified in plasma sample incubated without copper ion as shown in Fig. 3-A. Similar results were obtained in oxysteryl ester fraction.

The amounts of free and esterified forms of 7-ketocholesterol generated were increased with an increase of copper concentration (Fig. 4). Generated 7ketocholesterol level did not reach plateau up to a copper ion concentration of 2 . $0 \mathrm{mM}$. We chose a 2.0-mM concentration of copper ion to evaluate the copperdependent oxidative stress from the point of quantitative sensitivity and to measure produced oxysterols.

In the presence of $2.0 \mathrm{mM} \mathrm{CuSO}{ }_{4}(n=3), 497.1 \pm 187.9 \mathrm{ng}$ of free 7-ketocholesterol and $384.2 \pm 87.3 \mathrm{ng}$ of its ester form were produced (Table 2). As plasma levels of free and esterified cholesterol of these samples were $42.2 \pm 0.6 \mathrm{mg}$ /
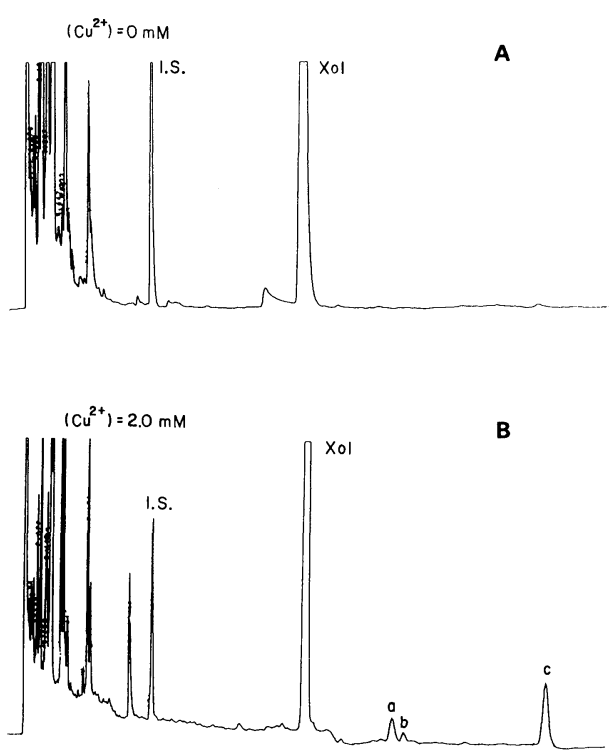

Fig. 3. Capillary GLC profiles of free fraction of oxysterols generated in the incubation without (A) and with $2 \mathrm{mM} \mathrm{Cu}^{2+}(\mathrm{B})$. Xol, cholesterol ; I.S., internal standard (5 $\beta$-cholestane); a, $7 \beta$-hydroxy-cholesterol ; b, 5, $6 \alpha$ epoxy-cholesterol ; c, 7-ketocholesterol. 


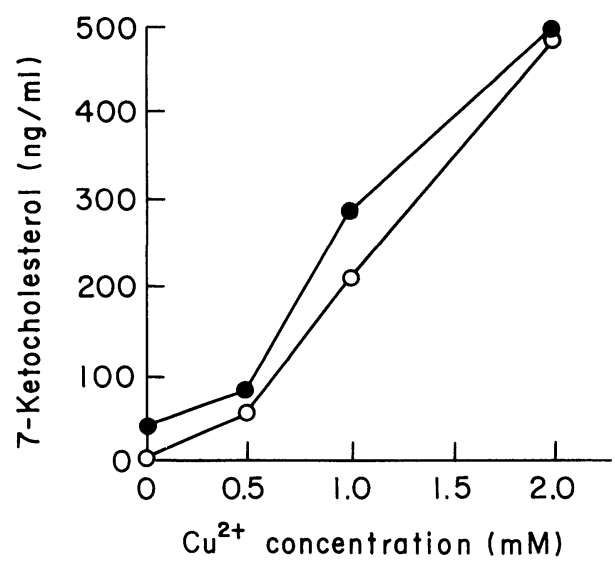

Fig. 4. Relationship between $\mathrm{Cu}^{2+}$ concentration and copper-mediated production of free (O) and esterified (๑) forms of 7-ketocholesterol. Free 7ketocholesterol and its ester were increased linearly with $\mathrm{Cu}^{2+}$ concentration from 0 to $2.0 \mathrm{mM}$.

TABLE 2. Levels of free and esterified forms of 7 -ketocholesterol generated in the presence of $\mathrm{CuSO}_{4}(2.0 \mathrm{mM})^{\mathrm{a}}$

\begin{tabular}{|c|c|c|c|c|c|}
\hline & $\begin{array}{c}\text { Plasma } \\
\text { cholesterol }\end{array}$ & $\begin{array}{c}\text { Generated } \\
\text { 7-ketocholesterol }\end{array}$ & \multicolumn{3}{|c|}{$\begin{array}{l}\text { Conversion rate } \\
\qquad\left(\times 10^{-3}\right)\end{array}$} \\
\hline & & & & mean士S.D. & \\
\hline \multirow[t]{3}{*}{ Free } & 42.02 & 482.5 & 1.15 & & \\
\hline & 41.61 & 734.2 & 1.76 & $1.19 \pm 0.44$ & \\
\hline & 43.06 & 274.6 & 0.68 & & \\
\hline \multirow[t]{3}{*}{ Ester } & 129.30 & 492.2 & 0.38 & & \\
\hline & 122.52 & 381.8 & 0.31 & $0.31 \pm 0.06$ & \\
\hline & 119.34 & 278.5 & 0.23 & & \\
\hline
\end{tabular}

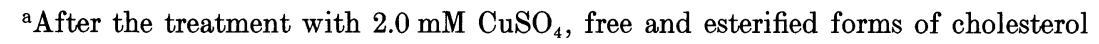
and 7-ketocholesterol were measured in triplicate.

${ }^{b}$ Values were corrected considering the mean recovery rate.

${ }^{* *} p>0.01$.

$100 \mathrm{ml}$ and $123.7 \pm 4.2 \mathrm{mg} / 100 \mathrm{ml}$, respectively, the conversion rate of cholesterol to 7-ketocholesterol was calculated as $(1.19 \pm 0.44) \times 10^{-3}$ for free form, and $(0.31 \pm$ $0.06) \times 10^{-3}$ for ester form (values were corrected considering the mean recovery rate). These results suggested that the 7 position of free cholesterol was more easily oxidized than that of esterified cholesterol $(p<0.01)$.

\section{Antioxidant effect of Probucol}

Probucol $(227 \mu \mathrm{M})$ inhibited the generation of 7-ketocholesterol in the pres- 
TABLE 3. Antioxidant effect of probucol on the copper-mediated oxidation of plasma cholesterol $^{\text {a }}$

\begin{tabular}{|c|c|c|c|c|}
\hline & \multicolumn{2}{|c|}{ 7-Ketocholesterol (ng/ml) } & \multirow{2}{*}{\multicolumn{2}{|c|}{ Inhibition $(\%)$}} \\
\hline & -Probucol & + Probucol & & \\
\hline & & & & mean \pm S.D. \\
\hline \multirow[t]{3}{*}{ Free } & 890.5 & 334.2 & 62.5 & \\
\hline & 585.0 & 348.4 & 40.4 & $54.7 \pm 12.4$ \\
\hline & 585.0 & 227.3 & 61.1 & \\
\hline \multirow[t]{3}{*}{ Esterified } & 618.7 & 197.2 & 68.1 & \\
\hline & 451.3 & 135.1 & 70.1 & $67.9 \pm 2.3$ \\
\hline & 451.3 & 155.8 & 65.5 & \\
\hline
\end{tabular}

${ }^{\text {aProbucol }}(227 \mu \mathrm{M})$ was added to $3 \mathrm{ml}$ of plasma and incubated with $2 \mathrm{mM} \mathrm{CuSO}_{4}$ in triplicate. Then, the generated free and esterified forms of 7-ketocholesterol were measured. The inhibition (\%) was calculated by comparing the levels of 7ketocholesterol produced with and without probucol.

ence of $2.0 \mathrm{mM} \mathrm{CuSO}$. The inhibition by probucol was $54.7 \% \pm 12.4 \%$ for the free oxysterol and $67.9 \% \pm 2.3 \%$ for the ester form (Table 3 ) ; and there was no significant difference between these two values.

\section{Discussion}

Hydroxylated derivatives of cholesterol are important intermediates of bile acids and steroid hormones (Björkhem 1985). In fact, hepatic and endocrine cells produce oxygenated sterol during cholesterol metabolism. Some oxysterols including 7-keto-, $7 \alpha-\mathrm{OH}-, 7 \beta-\mathrm{OH}-$, and $25-\mathrm{OH}$-cholesterols have been shown to be potent inhibitors of sterol synthesis due to the suppression of HMG-CoA reductase (Kandutsch and Chen 1973 ; Brown and Goldstein 1974). In cell culture experiments, these oxysterols are effective at a concentration of $10^{-9}$ to $10^{-6} \mathrm{M}$ (Kandutsch et al. 1978), and their bioactivity could be mediated by the specific binding protein in the cytosol (Taylor et al. 1984, 1988; Taylor and Kandutsch 1985).

To further elucidate the biological importance of oxysterols, it was necessary to develop a sensitive assay to meet the remarkably low levels of plasma. However, difficulties are caused by the close structural similarities and the large concentration difference of these compounds in comparison with cholesterol (Koopman et al. 1987).

Copper is widely described as a transition metal that generates hydroxy radicals. The reaction of copper complex with lipids generate a wide range of products, including epoxides, compounds containing the carbonyl group and volatile hydrocarbons. We also have to keep in mind that a number of oxygenated sterols are known to arise spontaneously from cholesterol by autooxidation under a wide variety of circumstances (Smith 1981; Breuer and Björkhem 1990).

We underwent copper-mediated oxidation of human plasma with a rather 
high concentration of copper ion. Generated free and ester form of oxysterols were extracted separately. Our finding showed that the 7 position of cholesterol was predominantly oxidized and 7-ketocholesterol was the main product in both froms. Small amounts of $7 \beta$-hydroxy- and 5, $6 \alpha$-epoxy-cholesterol were also identified.

We also showed that the cholesterol nucleus of the ester form (fatty-acylated) was more resistant against oxidative stress than that of the free form. We hypothesized that polyunsaturated fatty acids are very susceptible to oxidative attack (Esterbauer et al. 1987; Halliwell and Gutteridge 1989; Chahboun et al. 1990), and thereby protect the nucleus from the oxidation. Alternatively, the very hydrophobic esterified cholesterol occupies the core of plasma lipoprotein particles and this structural feature may be favorable to prevent the oxidation in the circulating blood. This resistance against oxidation stress of cholesteryl ester may be advantageous to cholesterol transport and storage in the human body.

Probucol consists of two butylated hydroxytoluene molecules connected by a sulfur-carbon-sulfur bridge. The phenolic-OH groups offer the molecule with some antioxidant activity. It is transported in plasma in the hydrophobic core of LDL and other lipoprotein particles (Marshall 1982). It has been already reported that the addition of probucol prevented endothelial cell-modified and cupric ion-catalyzed oxidation of LDL (Parthasarathy et al. 1986). We demonstrated that antioxidant effect of probucol was extended over cholesterol which is a prominent component of plasma LDL.

In conclusion, we developed a method to analyze free and esterified oxysterols, especially 7-ketocholesterol in human plasma and applied this method to evaluate copper-mediated oxidation of cholesterol. We found that the 7 position of cholesterol nucleus predominantly suffered from oxidation and that cholesterol nucleus of ester form was more resistant against oxidative stress than that of the free form. Probucol inhibited this oxidation of cholesterol. In the present study we used a large amount of $\mathrm{CuSO}_{4}$ and probucol, but sample separation and purification were enough to investigate oxysterols under more physiological conditions by using gas chromatography/mass spectrometry.

\section{References}

1) Assmann, G., Fredrickson, D.S., Sloan, H.R., Fales, H.M. \& Highet, R.J. (1975) Accumulation of oxygenated steryl esters in Wolman's disease. J. Lipid Res., 16, 2838.

2) Billheimer, J.F., Avart, S. \& Milani, B. (1983) Separation of steryl esters by reversed-phase liquid chromatography. J. Lipid Res., 24, 1646-1651.

3) Björkhem, I. (1985) Mechanism of bile acid biosynthesis in mammalian liver. In : Sterols and Bile Acids, edited by H. Danielsson \& J. Sjovall, Elsevier Science Publishers B.V., Amsterdam, pp. 231-278.

4) Breuer, O. \& Björkhem, I. (1990) Simultaneous quantification of several cholesterol autoxidation and monohydroxylation products by isotope-dilution mass spectrometry. Steroids, 55, 185-192. 
5) Brown, M.S. \& Goldstein, J.L. (1974) Suppression of 3-hydroxy-3-methylglutaryl Coenzyme A reductase activity and inhibition of growth of human fibroblasts by 7-ketocholesterol. J. Biol. Chem., 249, 7306-7314.

6) Brown, M.S. \& Goldstein, J.L. (1983) Lipoprotein metabolism in the macrophage: Implications for cholesterol deposition in atherosclerosis. Ann. Rev. Biochem., 52, 223-261.

7) Chahboun, S., Tallineau, C., Pontcharraud, R., Guettier, A. \& Piriou, A. (1990) Polyunsaturated fatty acid profiles and $\alpha$-tocopherol levels in plasma and whole blood incubated with copper. Evidence of inhibition of lipoperoxidation in plasma by hemolysate. Biochim. Biophys. Acta, 1042, 324-329.

8) Esterbauer, H., Jurgen, G., Quehenberger, O. \& Koller, E. (1987) Autoxidation of human low density lipoprotein: Loss of polyunsaturated fatty acids and vitamin $\mathrm{E}$ and generation of aldehydes. J. Lipid Res., 28, 495-509.

9) Halliwell, B. \& Gutteridge, J.M.C. (1989) Lipid peroxidation: A radical chain reaction. In: Free Radicals in Biology and Medicine, edited by B. Halliwell \& J.M. C. Gutteridge, Oxford University Press, New York, pp. 188-276.

10) Jialal, L., Freeman, D.A. \& Grundy, S.M. (1991) Varying susceptibility of different low density lipoproteins to oxidative modification. Arteriosclerosis Thromb., 11, 482488.

11) Kandutsch, A.K. \& Chen, H.W. (1973) Inhibition of sterol synthesis in cultured mouse cells by $7 \alpha$-hydroxycholesterol, $7 \beta$-hydroxycholesterol, and 7-ketocholesterol. J. Biol. Chem., 248, 8408-8417.

12) Kandutsch, A.K., Chen, H.W. \& Heiniger, H.J. (1978) Biological activity of some oxygenated sterols. Science, 201, 498-501.

13) Koopman, B.J., Van der Molen, J.C. \& Wolthers, B.G. (1987) Determination of some hydroxycholesterols in human serm samples. J. Chromatogr., 416, 1-13.

14) Lenz, M.L., Hughes, H., Mitchell, J.R., Via, D.P., Guyton, J.R., Taylor, A.A., Gotto, A.M., Jr. \& Smith, C.V. (1990) Lipid hydroperoxy and hydroxy derivatives in copper-catalyzed oxidation of low density lipoprotein. J. Lipid Res., 31, 1043-1050.

15) Marshall, F.N. (1982) Pharmacology and toxicology of probucol. Artery, 10, 7-21.

16) Parthasarathy, S., Young, S.G., Witztum, J.L., Pittman, R.C. \& Steinberg, D. (1986) Probucol inhibits oxidative modification of low density lipoprotein. J.Clin. Invest., 77, 641-644.

17) Smith, L.L. (1981) Biological effects. In: Cholesterol Autoxidation, edited by L.L. Smith, Plenum Press, New York, pp. 369-458.

18) Steinbrecher, U.P., Parthasarathy, S., Leake, D.S., Witztum, J.L. \& Steinberg, D. (1984) Modification of low density lipoprotein by endothelial cells involves lipid peroxidation and degradation of low density lipoprotein phospholipids. Proc. Natl. Acad. Sci., 81, 3883-3887.

19) Taylor, F.R. \& Kandutsch, A.A. (1985) Oxysterol binding protein. Chem. Phys. Lipid, 38, 187-194.

20) Taylor, F.R., Saucier, S.E., Shown, E.P., Parish, E.J. \& Kandutsch, A.A. (1984) Correlation between oxysterol binding to a cytosolic binding protein and potency in the repression of hydroxymethylglutaryl coenzyme A reductase. J. Biol. Chem., 259, $12382-12387$.

21) Taylor, F.R., Kandutsch, A.A., Anzalone, L., Phirna, S. \& Spencer, T.A. (1988) Photoaffinity labeling of the oxysterol receptor. J. Biol. Chem., 263, 2264-2269. 